\title{
Intracranial Mass Lesion Caused by Inflammatory Pseudotumor: A Case Report and Discussion
}

Andrew Reisner, $M D^{1,2^{*}}$, Matthew Schniederjan, $M D^{3,4}$, Joshua J Chern, $M D, P h D^{1,2}$, Andrew Appert, $B S^{5}$, Hannah E. Myers, BS. Psych ${ }^{5}$, Barunashish Brahma, MD ${ }^{1,2}$, Laura S Blackwell, PhD ${ }^{5}$, William $R$ Boydston, $M D, P h D^{2}$, and Sampath Prahalad, $M D^{1,6}$

${ }^{1}$ Department of Pediatrics, Emory University School of Medicine, USA

${ }^{2}$ Department of Neurosurgery, Emory University School of Medicine, USA

${ }^{3}$ Department of Pathology and Laboratory Medicine, Emory University

School of Medicine, USA

${ }^{4}$ Department of Pathology, Emory University School of Medicine, USA

${ }^{5}$ Department of Neuropsychology, Emory University School of Medicine, USA

${ }^{6}$ Section of Rheumatology, Children's Healthcare of Atlanta, USA

\begin{abstract}
Inflammatory pseudo tumors are uncommon lesions that may, on occasion, occupy the intracranial space. Their management is unstandardized. We present a case of skull-based inflammatory pseudotumor in a 14-year-old boy who presented with multiple cranial nerve palsies mimicking Gradenigo syndrome. This illustrative case and a review of the literature is presented to highlight noteworthy features of this rare condition. These include that inflammatory pseudotumor may be multifocal and biopsy of a more accessible, smaller lesion may prove diagnostic. Further, although surgery is an option, it is not always curative and often is associated with recurrences if no adjuvant treatment is given. In this case, surgical debulking of the larger skull base lesion was avoided and low-dose methotrexate treatment was not only well tolerated but produced 7-year remission. This case report may prove instructive to others managing this rare condition.
\end{abstract}

\section{Keywords}

Inflammatory pseudotumor, Central nervous system, Gradenigo syndrome, Methotrexate

\section{Introduction}

Inflammatory pseudotumor, also known as plasma cell granulomas, are rare lesions characterized pathologically by the combination of fibrosing and inflammatory elements $[1,2]$ in the absence of any explanation for their presence, making them in essence a diagnosis of exclusion. First described by Gleason and Busse in 1903, [3] but named by Umiker and Iverson [4] in 1954, inflammatory pseudotumor are now known to affect all organ systems, with an estimated $5 \%$ of cases involving the head and neck [5]. Their etiology remains unknown $[5,6]$.

Inflammatory pseudo tumors of the central nervous system (CNS) typically present with nonspecific symptoms and mass-related neurologic deficits [2]. The clinical, radiographic, and laboratory work-up of inflammatory pseudo tumors is most often nondiagnostic and diagnosis is typically made upon histopathologic examination [6]. Further, given the rarity of inflammatory pseudotumor of the CNS, ideal treatment strategies remain unclear. Regardless of treatment(s), recurrences are common [1].

We share the case of a 14-year-old boy who presented with pseudo-Gradenigo syndrome secondary to a clival and ipsilateral cavernous sinus tumor. Biopsy of a radiographically similar, smaller satellite lesion within the mastoid bone provided a diagnosis without the need for surgery on larger,

*Corresponding author: Andrew Reisner, MD, FACS, FAAP, Departments of Neurosurgery and Pediatrics, Emory University School of Medicine, Children's Healthcare of Atlanta 5455 Meridian Mark Dr. \#540, Atlanta, GA 30342, USA, Tel: 404-7852900, Fax: 404-785-2930

Accepted: May 02, 2021

Published online: May 04, 2021

Citation: Reisner A, Schniederjan M, Chern JJ, et al. (2021) Intracranial Mass Lesion Caused by Inflammatory Pseudotumor: A Case Report and Discussion. J Pediatr Neurol Neurosci 5(1):99-104 
pre-pontine and presenting lesion. The patient tolerated and responded well to methotrexate, with no evidence of recurrence at the 7-year follow-up.

\section{Case Presentation}

\section{History}

An otherwise healthy 14-year-old African American male presented with a 6-month history of gradual onset and progressively worsening, right-sided headaches. There was an associated 2-month history of worsening diplopia and ipsilateral facial numbness. There is a family history of sickle cell trait. There were no constitutional symptoms, such as fatigue or fever.

\section{Physical exam}

He had a right-sided complete sixth nerve and peripheral seventh palsy. There was no ipsilateral otitis media. Funduscopic exam, peripheral motor and sensory examinations were normal.

\section{Laboratory finding}

Normal values included a complete blood count, urinalysis, immunoglobin $\mathrm{G}$ subclasses, antinuclear antibody profile, antineutrophil cytoplasmic antibodies, rheumatoid factor, immunoglobulin G subclasses, lysosome and ACE. The comprehensive metabolic panel was within normal limits, except for an elevated ALT of 75 and AST of 42. The ESR was mildly elevated at 33. C3 and C4 were both elevated.

\section{Imaging studies}

CT scan showed a homogeneously enhancing mass involving the skull base and right temporal bone. There was mild partial opacification of the mastoid air cells and middle and
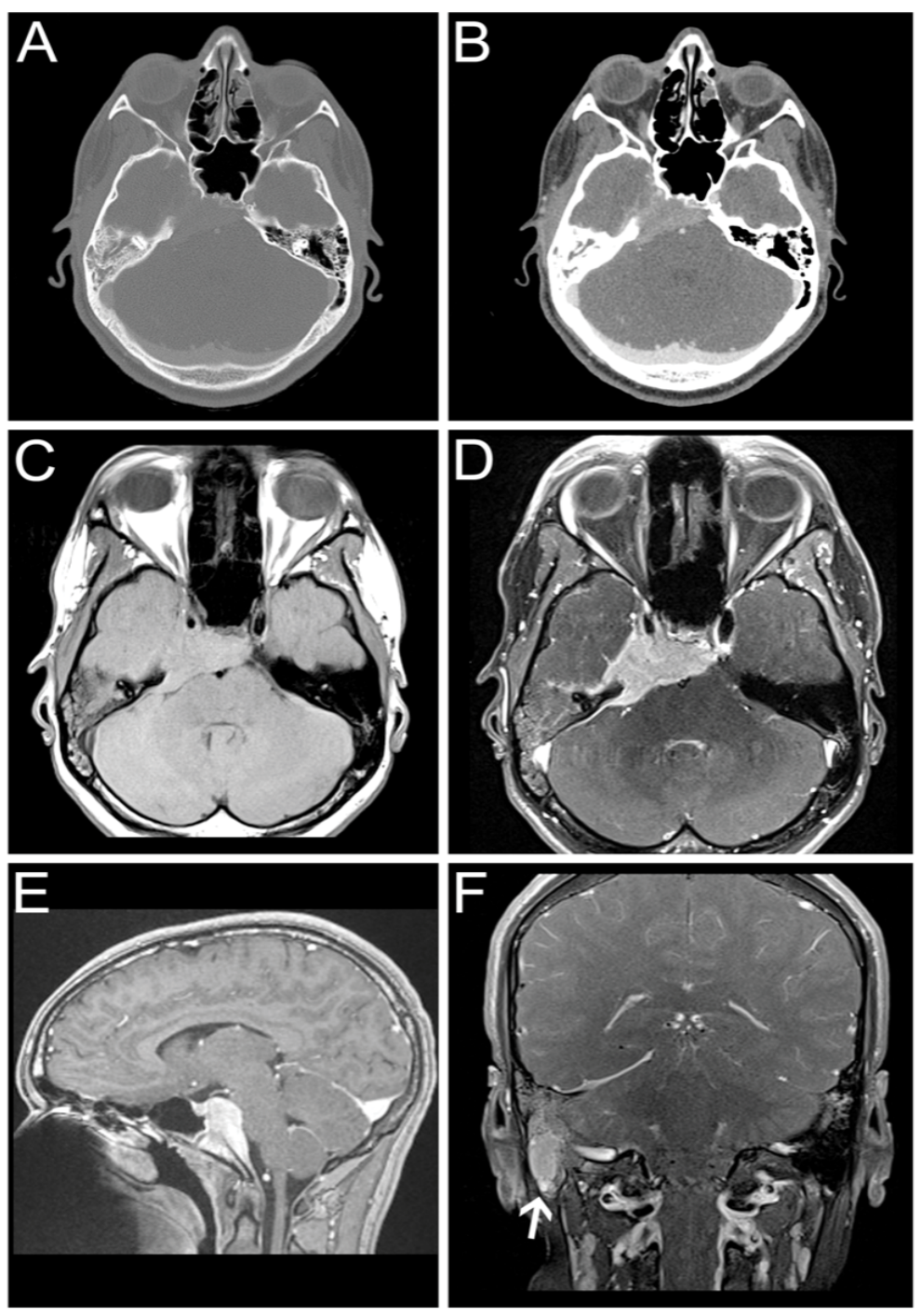

Figure 1: Presenting Radiographic Findings: Spiral axial scans of the head obtained from the base of the skull to the vertex after administration of contrast material demonstrate infiltrating soft tissue mass with bony destruction posterior to the right clivus with extension along the right side of the pons to the skull base (A). There is opacification of the right mastoid air cells, and mass effect upon the pons (B). Multiplanar, multisequence MR imaging of the brain performed on a 3 Tesla system with and without IV contrast. The mass is isointense on T1 (C) and T2 (D) imaging, with homogenous enhancement with contrast (E). A satellite lesion is noted at the temporal tip within the mastoid on the right ( $F$, arrow) with signal characteristics similar to the dominant mass. 
Citation: Reisner A, Schniederjan M, Chern JJ, et al. (2021) Intracranial Mass Lesion Caused by Inflammatory Pseudotumor: A Case Report and Discussion. J Pediatr Neurol Neurosci 5(1):99-104

inner ear on the right. The brain parenchyma and ventricles were normal (Figure $1 \mathrm{~A}$ and Figure $1 \mathrm{~B})$.

MRI revealed a homogeneously enhancing mass, predominantly isointense on T1 and T2 (Figure 1C and Figure 1D), involving the skull base and right temporal bone. It involved the clivus and sphenoid bone with extension into the cavernous sinus on the right. The mass extended into the prepontine cistern producing mild mass effect on the brainstem (Figure 1E).

Additionally, a soft tissue mass was noted within the right mastoid bone with signal characteristics of the dominant mass (Figure 1F). Fluid was present within the remainder of the mastoid air cells and middle cells on the right. Given the similarities of signal characteristics and enhancement of this lesion, there was a suspicion this represented a satellite lesion.

\section{Surgery}

The mastoid lesion was biopsied first by making an approximately $3 \mathrm{~cm}$ incision and dissecting the sternocleidomastoid muscle and decorticating the overlying bone. Frozen section pathology confirmed a nonneoplastic, possibly inflammatory lesion. Given that a diagnostic specimen had been obtained and that, based on the frozen section pathology it was unclear if surgery on the larger lesion was indicated, we elected not to proceed with the planned sub temporal approach to the clival and cavernous sinus lesion. The postoperative course was uneventful.

\section{Pathology}

Histologic sections showed a dense, polymorphous inflammatory infiltrate composed mostly plasma cells (Figure $2 A)$, with lesser components of lymphocytes, neutrophils, and histiocytes (Figure 2B), all in a background of reactive fibrosis. Rare eosinophils were present. The histiocytes identified by CD68 immunohistochemistry were not morphologically consistent with those of either Rosai-Dorfman or Langerhans cell histiocytosis, nor did they express S100 or CD1a by immunostaining (Figure 2C). Immunohistochemistry for ALK and smooth muscle actin was negative, essentially ruling out the diagnosis of inflammatory my fibroblastic tumor. Immunohistochemistry for IgG4 showed scattered individual IgG4-immunoreactive cells, less than $5 \%$ of the plasma cell infiltrate (Figure 2). In situ hybridization showed the plasma cells to be polytypic. Some extravasated sickled red blood cells were noted (Figure 2). The final diagnosis was inflammatory pseudotumor.

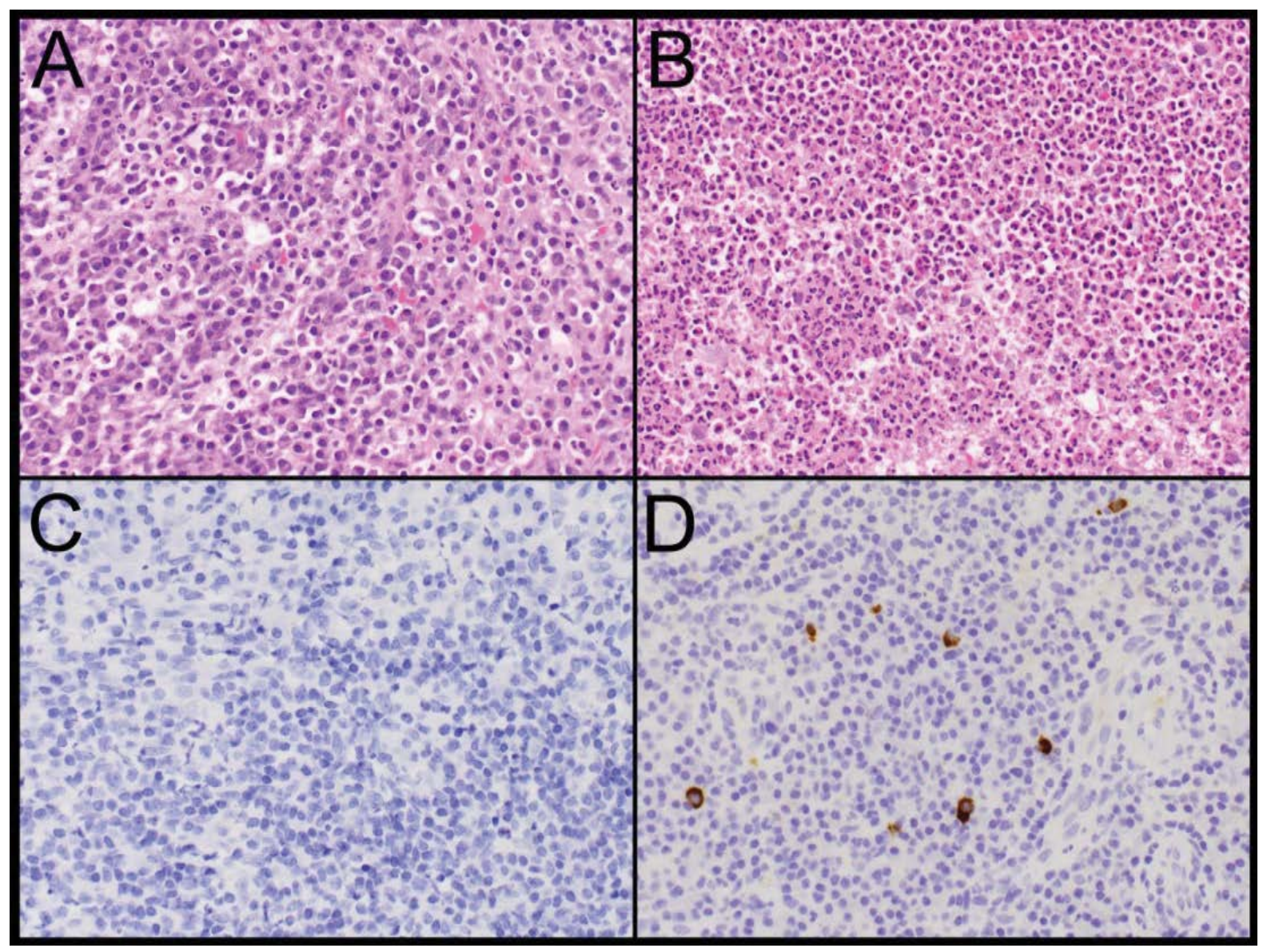

Figure 2: Histology: Histologic sections demonstrate a dense, polymorphous inflammatory infiltrate of plasma cells (A), with components of neutrophils, lymphocytes, and histiocytes (B). Immunostaining did not demonstrate CD1a (C) but was notable for scattered IgG4 positive cells ( $D$, brown). 

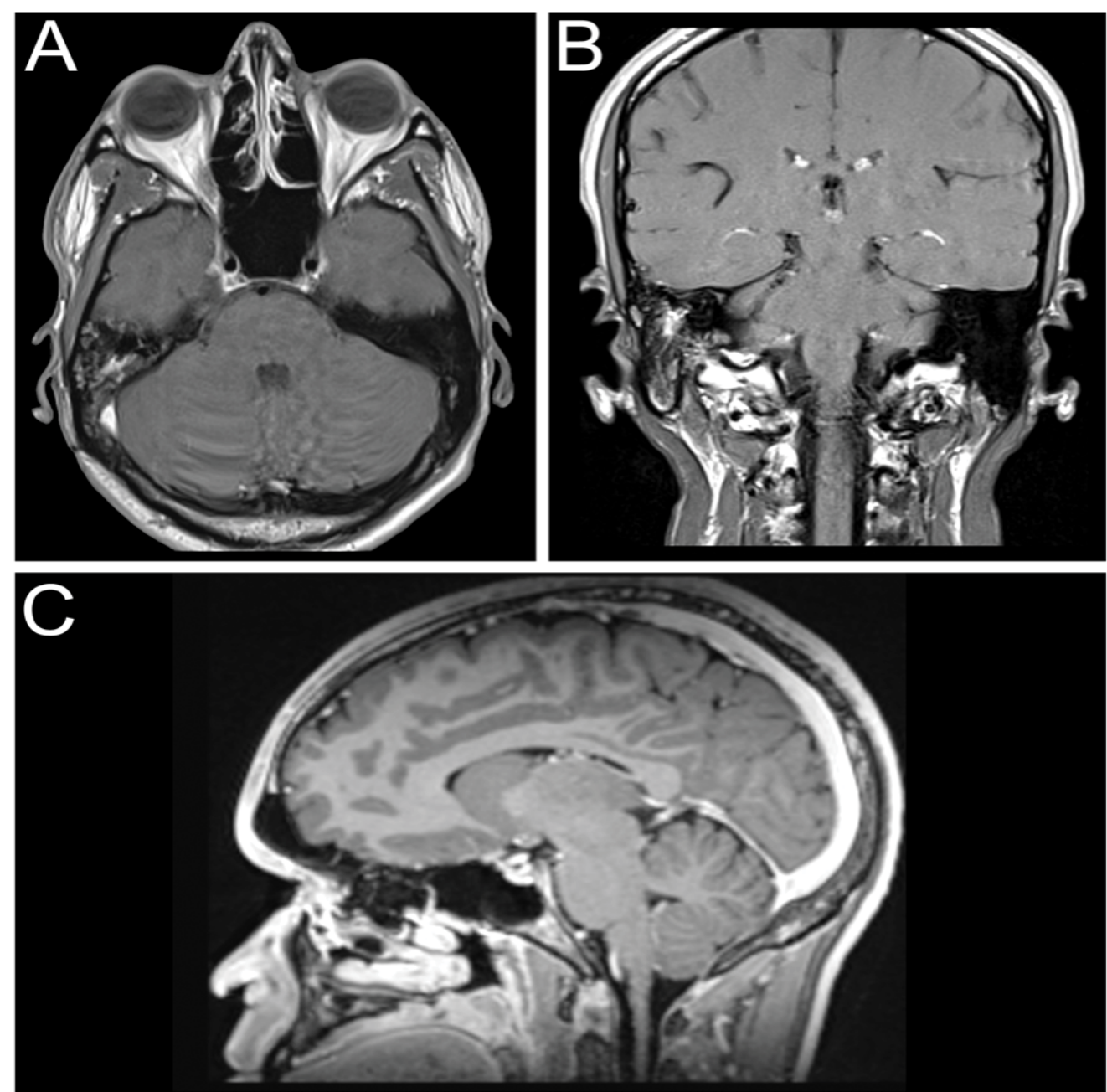

Figure 3: Post-treatment Radiography. Follow-up MRI obtained 5.5-years after diagnosis and treatment with methotrexate demonstrates interval resolution of the intracranial mass lesion $(A)$ and $(C)$, with stable postoperative changes of the right mastoid air cells (B).

\section{Treatment}

The patient was started on systemic corticosteroids, tapered over a month, and then referred to rheumatology. When seen in the rheumatology clinic, his presentation was determined consistent with an inflammatory pseudotumor. Laboratory evaluations argued against systemic lupus erythematosus or sarcoidosis. He was started on low-dose methotrexate $\left(9 \mathrm{mg} / \mathrm{m}^{2}\right)$ to avoid chronic steroid side effects. He was initially on subcutaneous methotrexate for 12 months at which point it was switched to oral administration. He continued methotrexate for the next 24 months, and since he had no recurrence based on clinical symptoms or MRI, methotrexate was tapered and discontinued 3-years after initiation of treatment.

\section{Follow-up}

At his 7-year follow-up, he was tumor free. His post-treatment MRI was normal (Figure 3).

\section{Discussion}

The diagnosis of intracranial inflammatory pseudotumor can be challenging. There are no pathognomonic clinical or imaging findings, and the diagnosis ultimately rests on histological characteristics [7]. The clinical presentation of intracranial inflammatory pseudotumor includes nonspecific constitutional signs and symptoms and mass related neurological findings $[2,7]$. This case presented as a pseudo-Gradenigo's syndrome [8]. Despite the finding of fluid in the middle ear, we do not believe the cranial nerve palsies were related to the otitis media and there was no concomitant retro-orbital pain ("classic" Gradenigo's syndrome). The sixth and seventh nerve palsies were due to the mass effect on the clival/cavernous sinus lesion on ipsilateral cranial nerves.

Similar to this case, radiological studies of intracranial inflammatory pseudotumors are often non-diagnostic. On CT, inflammatory pseudotumor may visualize with low, equal or high attenuation [6,7]. On occasion, they may be locally aggressive and demonstrate subjacent bone erosion. On MRI, inflammatory pseudotumor are T1-isointense or hypointense, T2-hypointense, and enhance with contrast [6,7]. Differentiation from neoplasia, infection or inflammatory lesions are not possible on imaging alone [7]. 
Histopathologically, inflammatory pseudotumor consist of myofibroblasts and chronic inflammatory cells with polyclonal immunoglobulin evidence $[2,6]$. The plasma cells are polyclonal, with evidence of mixed kappa and lambda light chain expression by in situ hybridization and immunohistochemistry. Inflammatory pseudotumor also have both $\mathrm{T}$ and B lymphocytes [9]. Histopathological findings in this case revealed chronic, focally acute inflammation, consistent with an inflammatory pseudotumor. Inflammatory myfibroblastic tumor, Langerhans histiocytosis, Rosai-Dorfman disease, xanthogranuloma, and sarcoidosis were excluded and there was no evidence of any infection.

Laboratory findings associated with inflammatory pseudotumor include anemia, hypergammaglobulinemia, increased erythrocyte sedimentation rate, leukocytosis, thrombocytosis, and increased prothrombin time. ${ }^{2}$ Among 18 patients with inflammatory pseudotumor of the CNS with available serum immunoglobulin results, eight (44\%) had polyclonal hypergammaglobulinopathy, while $3(17 \%)$ had high levels of a single immunoglobin detected [2]. The 14-year-old boy presented here showed no evidence of gammaglobulinopathy including IgG4 elevation.

Inflammatory pseudotumor involving the head and neck have been described in multiple locations [7]. A study of 40 cases of inflammatory intracranial lesions reported distribution of locations as predominately supratentorial (45\%), followed by intrasellar/suprasellar and cerebellar (each $n=$ $5,12.5 \%)$, brainstem $(10 \%)$, in the cerebellopontine angle (7.5\%), meningeal (7.5\%), and other locations (15\%) [10]. In this series, the lesion diameter ranged from 1 to $5.5 \mathrm{~cm}$ (mean, $2.6 \mathrm{~cm}$ ) [10]. Masses can attach to the parenchyma, dura, both, or intraventricular regions [2,9]. Inflammatory pseudotumor can present as a single or multiple mass $[2,8]$.

Diagnostic andor therapeutic surgery is usually indicated in cases of inflammatory pseudotumor of the head and neck, including intracranial lesion. Resection of well localized masses may be curative $[2,9,10]$. However, the utility of radical surgical approaches for more extensive lesions are being reevaluated due to increased awareness of tumor recurrence, slow progression of natural history and recognition of alternate, non-surgical therapies $[6,7,9]$. In a recent study of 40 patients with an inflammatory intracranial lesion treated over a 10-year period, 17 patients underwent surgical removal of the mass lesion, while only a biopsy was taken in 23 patients [10]. Overall, surgery was judged as valuable in 35 patients (87.5\%) [10]. In the presented case, biopsy of an accessible satellite lesion was adequate for diagnostic purposes and permitted consideration of medical treatments.

Steroid therapy, radiotherapy, and immunosuppressive therapy are treatment options for inflammatory pseudotumor $[1,6,9]$. Initial response rate to steroids has been reported to be high, reaching approximately $80 \%$ [11]. There are published cases in which monoclonal antibodies (Rituximab) and immunosuppressants (mycophenolate mofetil) have been used on guidance from rheumatologists [6]. There are a few case reports of methotrexate being used for the treatment of pseudotumor, including intracranial use in a pediatric patient with recurrent disease [12-14].
In order to minimize toxicity associated with chronic steroid use, methotrexate was prescribed. Methotrexate is a safe, disease-modifying antirheumatic drug used to treat a variety of inflammatory disorders. It is given once a week by oral or subcutaneous administration and requires safety labs every 12 weeks.

In this case, the patient responded well to, and tolerated prolonged methotrexate with no evidence of recurrence at 7-years after diagnosis and approximately 4-years after discontinuation of all treatments.

\section{Conclusion}

We present a case of intracranial inflammatory pseudotumor in a 14-year-old boy who presented with pseudo-Gradenigo syndrome. Highlights of this case and a literature review that may prove instructive to others managing this rare condition include 1) Pseudotumor can be multifocal and biopsy of smaller and more accessible lesions may prove diagnostic; 2) Surgical debulking is an option, but it is not always curative and is associated with recurrences if no adjuvant treatment is given; and 3) Steroids followed by methotrexate treatment may induce remission.

\section{Disclosures}

Dr. Reisner is supported in part by the Elaine and John C. Carlos Chair Fund and D. Prahalad is supported in part by the Marcus Foundation, Inc."

\section{Conflict of Interest}

None.

\section{References}

1. Häusler M, Schaade L, Ramaekers VT, et al. (2003) Inflammatory pseudo tumors of the central nervous system: Report of 3 cases and a literature review. Hum Pathol 34: 253-262.

2. Tresser N, Rolf C, Cohen M (1996) Plasma cell granulomas of the brain: Pediatric case presentation and review of the literature. Childs Nerv Syst 12: 52-57.

3. Gleason J (1903) Idiopathic myositis involving the extraocular muscles. Opthalmol Rec 12: 471-478.

4. Umiker WO, Iverson L (1954) Post inflammatory tumors of the lung; Report of four cases simulating xanthoma, fibroma, or plasma cell tumor. J Thorac Surg 28: 55-63.

5. Coffin CM, Watterson J, Priest JR, et al. (1995) Extrapulmonary inflammatory myofibroblastic tumor (inflammatory pseudotumor). A clinicopathologic and immunohistochemical study of 84 cases. Am J Surg Pathol 19: 859-872.

6. Ortlip TE, Drake VE, Raghavan P, et al. (2017) Inflammatory pseudotumor of the temporal bone: A case series. Otol Neurotol 38: 1024-1031.

7. Lai LM, McCarville MB, Kirby $P$, et al. (2015) Shedding light on inflammatory pseudotumor in children: Spotlight on inflammatory myofibroblastic tumor. Pediatr Radiol 45: 1738-1752.

8. Pedroso JL, de Aquino CC, Abrahão A, et al. (2011) Gradenigo's syndrome: Beyond the classical triad of diplopia, facial pain and otorrhea. Case Rep Neurol 15: 45-47.

9. Patnana M, Sevrukov AB, Elsayes KM, et al. (2012) Inflammato- 
ry pseudotumor: The great mimicker. AJR Am J Roentgenol 198: W217- W227.

10. Al-Afif S, Hatipoglu Majernik G, Al Krinawe Y, et al. (2019) The role of neurosurgery in the treatment of intracranial tumor-like inflammatory lesions. World Neurosurg 124: e81-e95.

11. Prabhu RS, Kandula S, Liebman L, et al. (2013) Association of clinical response and long-term outcome among patients with biopsied orbital pseudotumor receiving modern radiation therapy. Int J Radiat Oncol Biol Phys 85: 643-649.
12. Chong S, Carren, Shashinder S, et al. (2014) Aggressive inflammatory pseudotumor of the maxillary sinus and orbit. Ear Nose Throat J 93: 108-111.

13. Smith JR, Rosenbaum JT (2001) A role for methotrexate in the management of non-infectious orbital inflammatory disease. $\mathrm{Br}$ J Ophthalmol 85: 1220-1224.

14. Shah MD, McClain KL (2005) Intracranial plasma cell granuloma: Case report and treatment of recurrence with methotrexate and 6-mercaptopurine. J Pediatr Hematol Oncol 27: 599-603. 